\section{Anexon's fail-safe BNP}

\section{By Kai-Jye Lou, Staff Writer}

Researchers at the Mayo Clinic and University of Minnesota have identified an alternatively spliced form of the hormone B-type natriuretic peptide that lacks the blood pressure-lowering effects that have curtailed the use of its regular form in heart failure patients. ${ }^{1}$ Later this year, Anexon Inc. plans to start Phase I testing for the splice variant ANX-042, one in the family of BNP splice variants from the Mayo Clinic that the company has exclusively licensed.

Natriuretic peptide precursor B (NPPB; BNP), also known as B-type natriuretic peptide, is a peptide hormone normally secreted by ventricular cardiomyocytes. The peptide helps clear salt and water from the body and also acts as a vasodilator to reduce blood pressure. ${ }^{2}$ During heart failure, the body tries to compensate for decreased cardiac output by increasing expression of BNP.

Thus, there was a clear therapeutic rationale for development of Scios Inc.'s Natrecor nesiritide recombinant $\mathrm{BNP}$, which received approval in 2001 to treat acutely decompensated congestive heart failure in patients with dyspnea at rest or with a small amount of activity. Sales of the drug ramped up quickly and prompted

Johnson \& Johnson to acquire Scios in 2003 for $\$ 2.4$ billion. $^{3}$

The peptide, however, had a narrow therapeutic window because its potent blood pressure-lowering effects can lead to hypotension and impaired renal function. By 2005, Natrecor use was associated with kidney problems and mortality. ${ }^{4}$ As a result, a panel of clinicians put together by J\&J recommended limiting the use of the drug to hospitalized patients. ${ }^{5}$

To address the safety issues associated with Natrecor, a research group led by Robert Simari embarked on a search for endogenous BNP variants. The team's RNA isolation and reverse transcription studies led to the discovery of an alternatively spliced variant that retained the peptide's renal-protective effects but lacked significant vasoactive effects.

Simari's group truncated the 60-amino-acid splice variant down to 42 amino acids to help facilitate large-scale synthesis. "We identified a peptide that increased diuresis, natriuresis and glomerular filtration rates" - which are measures of renal function-without blood pressure-lowering effects, said Simari, a cofounder of Anexon and a professor of medicine at the
Mayo Clinic. "This peptide seems to dissociate the vasoactive effects from the beneficial renal effects of BNP."

In a canine model of heart failure, i.v. infusion of the nonvasoactive BNP increased the glomerular filtration rate, urinary sodium excretion and urine flow compared with baseline. A sustained i.v. infusion of the highest dose of the peptide ( $100 \mathrm{pmol} / \mathrm{kg}$ per minute) did not significantly affect mean arterial blood pressure in five dogs.

Results were published in the Proceedings of the National Academy of Sciences.

"People have always hypothesized that such an exclusive renal-focused peptide would exist-scientists have been looking for such a peptide for about 15 years, and now this group has discovered it," said Steve Landau, a director at Healthcare Ventures LLC, which has provided an undisclosed amount of initial venture financing for Anexon.

\section{Expanding BNP}

Anexon hopes that ANX-042's profile will allow the company not only to address a larger segment of the heart failure population than Natrecor, but also to make inroads into other therapeutic areas.

"We believe this peptide might be able to protect the kidney and preserve or improve kidney function during acute heart failure," Simari told SciBX. "Its renal-protective effects could allow patients to be treated more safely and spend less time in the hospital," because the sooner a heart failure patient is stabilized, the better the outcome is likely to be.

"We know that the glomerular filtration rate is a primary determinant of morbidity and mortality in patients with heart failure," he added.

"Use of Natrecor was limited primarily to about $10 \%$ of hospitalized patients with heart failure," noted Landau. "With this alternatively spliced peptide, we are looking at potentially being able to benefit up to $60 \%$ of the hospitalized patient population in heart failure."

For example, he thinks ANX-042 could be used in more heart failure patients, including those that have low blood pressure and abnormal renal function, in patients with renal damage that develop heart failure or in patients with or at risk of renal damage.

Simari thinks the nonvasoactive BNP also could have utility outside of heart failure. He noted that the peptide could potentially show benefit in patients with acute renal dysfunction caused by cardiovascular surgery or contrast infusion.

"Our first goal is to protect the kidney in the setting of acute heart failure and then branch out to other disease areas," Simari said.

\section{Pressure's on}

In the heart failure arena, ANX-042 would be unlikely to fully replace Natrecor because the latter's blood pressure-lowering effects could be desirable in certain cases.

Heart failure patients who are already taking angiotensin-converting 
enzyme (ACE) inhibitors or diuretics may not want the blood pressure-lowering effects of nesiritide, said Peter Liu, scientific director of the Canadian Institutes of Health Research's Institute of Circulatory and Respiratory Health and a professor of medicine at the University of Toronto. In those cases, he said, use of the nonvasoactive BNP variant may be preferred.

On the other hand, Liu told SciBX, "if a patient with heart failure and high blood pressure comes in with some mild kidney compromise, the kidneys could be shutting down because the renal blood vessels are too constricted." In that case, a compound like ANX-042 that lacks vascular effects might not be able to manifest its renal-protective effects.

Indeed, Hsiao Dee Lieu thinks the nonvasoactive, alternatively spliced BNP would probably be better suited for use in acute heart failure patients with low blood pressure. Lieu is VP of clinical research at Nile Therapeutics Inc. and an assistant clinical professor of medicine at the University of California, San Francisco.

Nile's lead compound is CD-NP, a chimeric natriuretic peptide that selectively agonizes both natriuretic peptide receptor A (NPR1; NPRA) and NPR2 (NPRB). The compound is expected to enter a Phase IIb trial in acute decompensated heart failure patients this quarter.

Lieu said he also wanted to better understand the mechanism of action for the nonvasoactive peptide's renal-protective effects. He noted that data in the PNAS paper showed that the peptide had less activity toward the natriuretic peptide receptors than normal BNP.

"The most important question to answer is: Is this configuration of the peptide, at the end of the day, going to be able to maintain a clinical ben- efit?" said the University of Toronto's Liu. "Because heart failure patients come from a variety of backgrounds, it is important to determine whether the peptide will yield clinical benefit in different heart failure settings."

Simari agreed. "Almost certainly, patients will not become hypotensive, but the real key is whether the compound will provide enough support for the patients and improve patient outcomes," he said.

Mayo Clinic has a pending patent that covers the alternatively spliced BNP. Mayo Clinic also holds an equity position in Anexon.

Lou, K.-J. SciBX 2(26); doi:10.1038/scibx.2009.1028

Published online July 9, 2009

\section{REFERENCES}

1. Pan, S. et al. Proc. Natl. Acad. Sci. USA; published online June 15 , 2009; doi:10.1073/pnas.0811851106

Contact: Robert D. Simari, Mayo Clinic, Rochester, Minn. e-mail: simari.robert@mayo.edu

2. Woodard, G.E. \& Rosado, J.A. J. Cell Mol. Med. 11, 1263-1271 (2007)

3. Pierce, E. \& Brown, S. BioCentury 11(8), A14-A15; Feb. 17, 2003

4. Jonathan D. et al. JAMA 293, 1900-1905 (2005)

5. Bernstein, K. BioCentury 13(38), A6-A10; Aug. 29, 2005

\section{COMPANIES AND INSTITUTIONS MENTIONED}

Anexon Inc., Cambridge, Mass.

Canadian Institutes of Health Research, Toronto, Ontario, Canada Healthcare Ventures LLC, Cambridge, Mass.

Johnson \& Johnson (NYSE:JNJ), New Brunswick, N.J.

Mayo Clinic, Rochester, Minn.

Nile Therapeutics Inc. (NASDAQ:NLTX), Berkeley, Calif.

University of California, San Francisco, Calif

University of Minnesota, Minneapolis, Minn.

University of Toronto, Toronto, Ontario, Canada 